\title{
Gastropleural fistula from gastric perforation due to renal cell carcinoma after bevacizumab chemotherapy: a case report
}

\author{
Olívia Meira Dias,' Caroline Chaul de Lima Barbosa," Lisete Ribeiro Teixeira,' Francisco S. Vargas' \\ 'Pulmonology Department, Heart Institute (INCOR) - Hospital das Clínicas da Faculdade de Medicina da Universidade de São Paulo, São Paulo/SP, Brazil. \\ "Oncology Department, São Paulo State Cancer Institute (ICESP) - São Paulo/SP, Brazil.
}

Email: meiradias@yahoo.com.br

Tel.: 5511 3442-0010

\section{CASE HISTORY}

A 39-year-old female was admitted for antibiotic treatment of sepsis of unknown origin, which had been empirically treated with piperacillin-tazobactam. The patient had previously (five months prior) been diagnosed with renal cell carcinoma with clear cell histological features and had undergone a left partial nephrectomy without excision of the entire tumor. Three months before admission, the patient was recruited to participate in a clinical trial investigating hepatic and pancreatic metastasis; the participants received interferon and bevacizumab in one of the protocols. The patient did not present any indication of pulmonary involvement from cancer in a tomography scan taken one month before admission.

During her hospital stay, the patient presented a sudden onset of shortness of breath, tachypnea, and chest discomfort without hemodynamic repercussion. On physical examination, the patient exhibited decreased breath sounds in her left hemithorax and tympanic percussion. Trachea deviation was also observed to the right and the patient presented with bilateral jugular stasis. Her saturation was 91\% despite oxygen given at a $10-1 / \mathrm{min}$ rate through an oxygen mask. A thoracocentesis with air escape in the second intercostal space was immediately performed by the thoracic surgeon. A left-sided hydropneumothorax was diagnosed in the chest roentgenogram with contralateral mediastinal shift (Figure 1). The patient was transferred to the intensive care unit (ICU) with chest discomfort despite drainage with a chest drain. A small amount of odorless, turbid brownish fluid came from the pleural space just after the drainage.

After non-invasive mechanical ventilation, the chest discomfort progressively improved, and both lungs were expanded. Upon discharge from the ICU, a liquid resembling nasogastric feed started to emerge from the chest drain in increasing amounts and shortly after commencing feeding with an output of more than $2300 \mathrm{ml}$ per day. Biochemical analysis of the pleural effusion revealed a neutrophilic exudate with a low $\mathrm{pH}$ (6.32), low protein $(1.5 \mathrm{~g} / \mathrm{dl})$, normal glucose $(103 \mathrm{mg} / \mathrm{dl})$, and high levels of

Copyright (c) 2011 CLINICS - This is an Open Access article distributed under the terms of the Creative Commons Attribution Non-Commercial License (http:// creativecommons.org/licenses/by-nc/3.0/) which permits unrestricted noncommercial use, distribution, and reproduction in any medium, provided the original work is properly cited. lactate dehydrogenase (DHL) and amylase (16864 U/1 and $873 \mathrm{U} / 1$, respectively). The level of serum amylase was $23 \mathrm{U} / \mathrm{l}$. The cytological exam was inconclusive. Because of the high levels of amylase, an esophageal perforation was initially suspected. A methylene blue test was performed at the bedside with a positive result, indicating leakage of this substance in the chest drain insertion after oral administration. Computed tomography with oral contrasted medium through the feeding tube showed a gastropleural fistula originating from the greater curvature and extending to the left subphrenic space (Figure 2). The patient was submitted to parenteral nutrition.

Two days after the diagnosis, the patient presented sudden bleeding exteriorized by the chest tube (approximately $1800 \mathrm{ml}$ of sanguineous pleural effusion) and hypovolemic shock. The patient was transferred again to the ICU where the patient received fluid, blood, and vasopressor support and recovered from the shock. Upper gastrointestinal endoscopy revealed a large blood clot in the greater curvature and fundus, which impeded further exploration. The patient was submitted to embolization of the splenic arteries in the interventional radiology service with success, and no further bleeding episodes occurred. After discussion with the Gastro Surgery team and considering the surgical risk due to the patient's poor status, the patient was transferred to a palliative care facility. The patient died ten days after the embolization, due to shock and acute respiratory insufficiency.

\section{DISCUSSION}

Gastropleural fistula is an uncommon diagnosis. It has been previously reported as a complication after pulmonary resections, ${ }^{1}$ trauma (especially due to traumatic diaphragmatic hernia), complications of peptic ulcer disease, and malignancy. ${ }^{2}$ Some case reports have indicated a predisposition caused by oral steroids or anti-inflammatory drug intake when the cause is gastric perforation. ${ }^{3}$ According to a recent literature review, ${ }^{4}$ subphrenic pathologies less frequently lead to this condition, generally due to diaphragmatic erosion. Supradiaphragmatic conditions, such as infections, intrathoracic surgeries, and fistulas due to procedures (caused by forceful intercostal tube insertion ${ }^{4}$ and after nasogastric tube placement after gastric adenocarcinoma resection) $^{5}$ can also result in this condition.

Gastric ulcers have been described as the most frequent cause of gastropleural fistulas. With the advent of intensive 


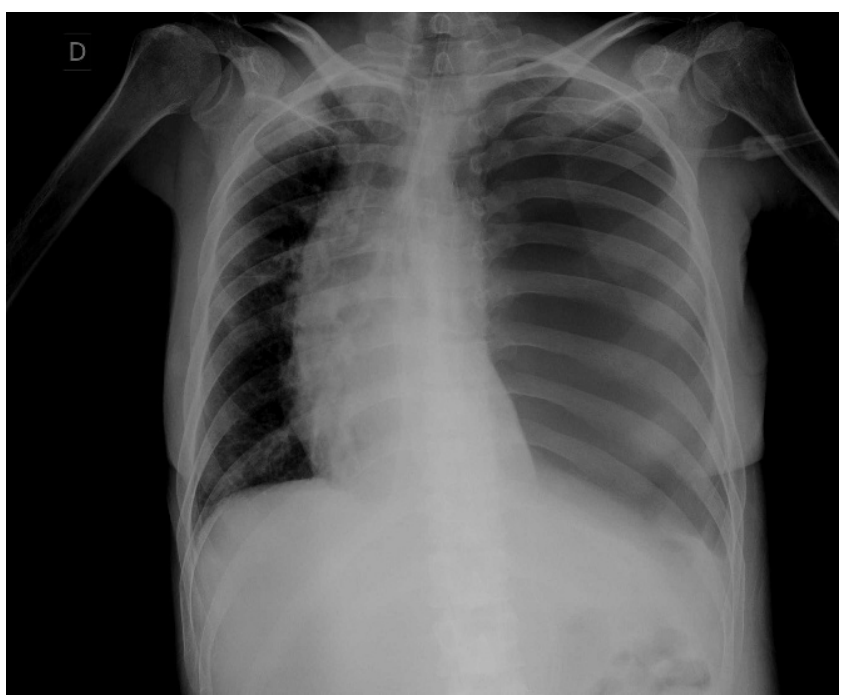

Figure 1 - Chest roentgenogram showing left-sided tension hydropneumothorax with contralateral mediastinal shift.

treatment regimens involving proton pump inhibitors and eradication therapy for Helicobacter pylori infection, one would expect that the incidence of gastropleural fistulas would decrease. ${ }^{6}$ Unfortunately, gastropleural fistulas can also occur secondarily to intra-abdominal or thoracic malignancies, which occur increasingly frequently with aging. Published case reports include a B-cell lymphoma ${ }^{7}$ and two gastric mucosa-associated lymphoid tissue (MALT) lymphomas. 6,8

To our knowledge, this is the first reported case of gastropleural fistula caused by gastric perforation due to renal cell carcinoma invasion. Bevacizumab is a recombinant, humanized monoclonal antibody that binds to vascular endothelial growth factor (VEGF) and inhibits angiogenesis. VEGF is a survival factor for blood vessels in certain types of tumors. Inhibition of VEGF signaling in tumors results in the cessation of angiogenesis, regression of some tumor vessels, normalization of others, and improved drug delivery. Unlike the blood vessels in tumors, the normal vasculature in adults is considered to be stable and, therefore, largely independent of VEGF for survival. However, VEGF and VEGF receptors are still highly expressed in several adult organs, and endothelial fenestrations can be induced by VEGF inhibitors in vitro and in vivo. ${ }^{9}$ The inhibition of VEGF signaling can eliminate fenestrations in tumor vessels, leading to impaired wound repair and predisposing the patient to fistula formation and bleeding. This potent anti-neoplastic agent has recently been reported to be associated with an increased risk of wound suture dehiscence requiring intervention, fistulas, gastrointestinal perforation, and serious bleeding/ hemorrhage. ${ }^{10}$ Among the nongastrointestinal fistulas, tracheoesophageal, bronchopleural, biliary, vaginal, renal, and bladder fistulas have been observed, most commonly within the first six months of treatment. ${ }^{11}$ No cases of gastropleural fistulas have been reported.

The diagnosis of gastropleural fistula is usually determined by contrast radiology, ${ }^{12}$ upper gastrointestinal endoscopy, and pleural fluid testing. In the present case, the cytological exam was not helpful, due to cellular lysis. Although conservative management has been suggested in a recent review of enteral fistulas after bevacizumab chemotherapy, ${ }^{11}$ this approach is often unsuccessful in patients with gastropleural fistulas, making surgery mandatory. Unfortunately, the potential bleeding caused by bevacizumab would impose unacceptable risks to this patient if surgery was attempted.

In conclusion, although gastropleural fistulas are relatively uncommon, the condition requires prompt surgical intervention. Although its incidence due to gastric perforations is low, subdiaphragmatic and intrathoracic tumors will increase the likelihood of this complication. New chemotherapeutic agents, such as bevacizumab, can promote fistulas caused by tumor necrosis, and gastropleural fistulas should be considered as a possible complication.
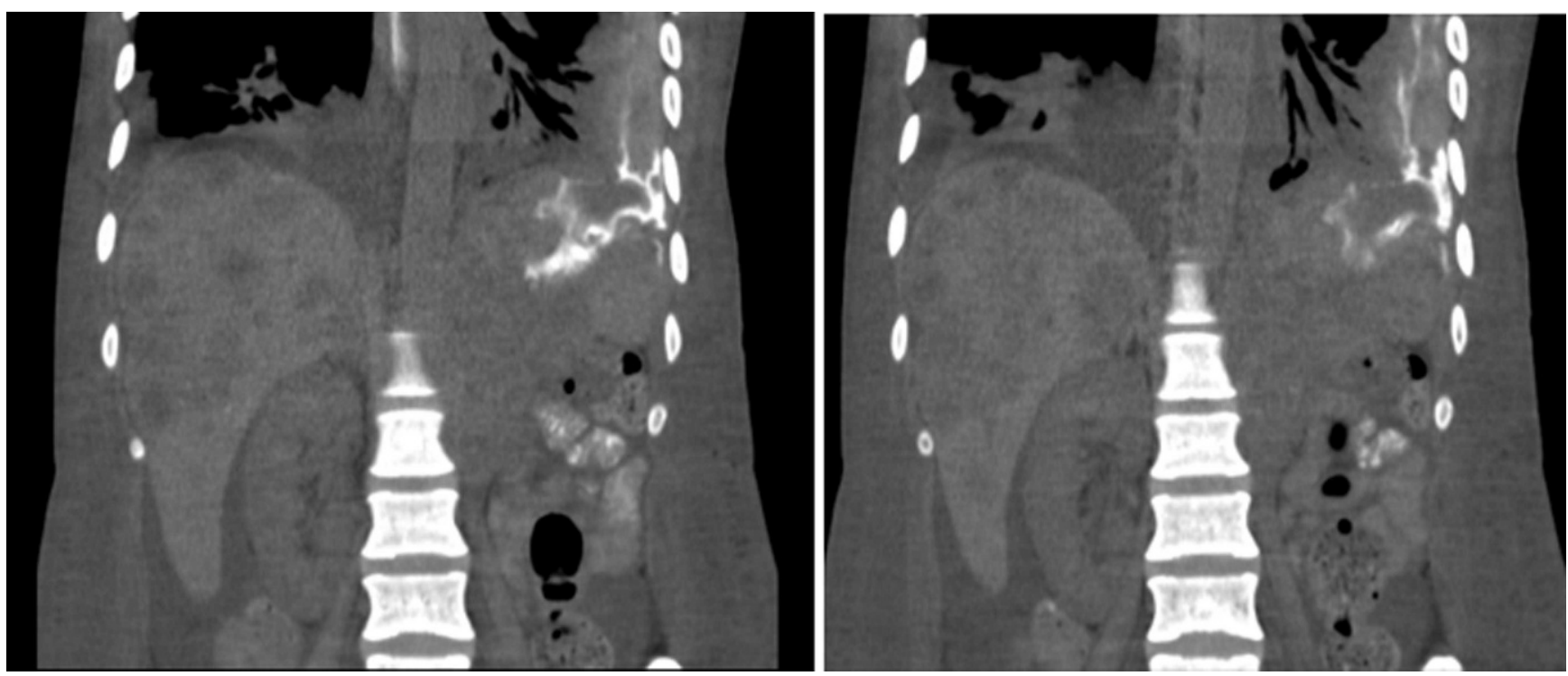

Figure 2 - Coronal computed tomography reconstruction with oral contrasted medium through the feeding tube. A heterogeneous solid mass with extensive necrotic areas in the left renal space invades the posterior wall of the stomach. The gastropleural fistula originates from the greater curvature extending to the left subfrenic space. Bilateral pleural effusion and partial athelectasis from the left lower lobe with air bronchograms are observed. Other small hipoattenuating hepatic lesions can also be seen due to hepatic metastasis. 


\section{REFERENCES}

1. Takeda S, Funaki S, Yumiba T, Ohno K. Gastropleural fistula due to gastric perforation after lobectomy for lung cancer. Interact CardioVasc Thorac Surg. 2005;4:420-2, doi: 10.1510/icvts.2005.108779.

2. Schwab RJ, Jarvik JG. Tension pneumothorax secondary to a gastropleural fistula in a traumatic diaphragmatic hernia. Chest. 1991;99:247-9, doi: 10.1378/chest.99.1.247.

3. Keefe P, Goldstraw P. Gastropleural fistula following pulmonary resection. Thorax. 1993;48:1278-9, doi: 10.1136/thx.48.12.1278.

4. Darbari A, Tandon S, Singh GP. Gastropleural fistula: rare entity with unusual etiology. Ann Thorac Med. 2007;2:64-5, doi: 10.4103/1817-1737. 32233.

5. Bini A, Grazia M, Petrella F, Stella F, Bazzocchi R. Spontaneous biliopneumothorax (Thoracobilia) following gastropleural fistula due to stomach perforation by nasogastric tube. Ann Thorac Surg. 2004;78:339-41, doi: 10.1016/S0003-4975(03)01282-7.

6. Adachi Y, Sato Y, Yasui H, Nishimura S, Tanimura A, Yuasa H, et al. Gastropleural fistula derived from malignant lymphoma. J Gastroenterol. 2002;37:1052-6, doi: 10.1007/s005350200177.
7. Mehran A, Ukleja A, Szomstein S, Rosenthal R. Laparoscopic partial gastrectomy for the treatment of gastropleural fistula. JSLS. 2005;9:213-5.

8. Warburton CJ, Calverly PM. Gastropleural fistula due to gastric lymphoma presenting as tension pneumothorax and empyema. Eur Resp J. 1997;10:1678-9, doi: 10.1183/09031936.97.10071678.

9. Kamba T, Tam BY, Hashizume H, Haskell A, Sennino B, Mancuso MR, et al. VEGF-dependent plasticity of fenestrated capillaries in the normal adult microvasculature. Am J Physiol Heart Circ Physiol. 2006;290: 560-76, doi: 10.1152/ajpheart.00133.2005.

10. Spigel DR, Hainsworth JD, Yardley DA, Raefsky E, Patton J, Peacock N, et al. Tracheoesophageal fistula formation in patients with lung cancer treated with chemoradiation and bevacizumab. J Clin Oncol. 2010;28:438, doi: 10.1200/JCO.2009.24.7353.

11. Badgwell BD, Camp ER, Feig B, Wolff RA, Eng C, Ellis LM et al. Management of bevacizumab-associated bowel perforation: a case series and review of the literature. Ann Oncol. 2008;19:577-82, doi: 10.1093/annonc/mdm508.

12. Chow H, Jung A, Talbott J, Lin AM, Daud AI, Coakley FV. Tumor fistulization associated with targeted therapy: computed tomographic findings and clinical consequences. J Comput Assist Tomogr. 2011;35: 86-90, doi: 10.1097/RCT.0b013e3181fce2cb. 